\title{
Morphologies of particles deposit on a single fiber due to the inertia interception
}

\author{
Hui Zhu ${ }^{1, a}$, Can $\mathrm{Qi}^{2, \mathrm{~b}}$, Haiming $\mathrm{Fu}^{3, \mathrm{c}}$, Yanming Kang ${ }^{4, \mathrm{~d}}$ \\ 1,2, Department of Energy Engineering and Building Environment, Guilin University of Aerospace \\ Technology, Guilin 541004, China \\ ${ }^{3,4}$ School of Environmental Science and Engineering, Donghua University, Shanghai 200051, China \\ azhuhui@guat.edu.cn, ${ }^{\text {b }}$ qican@guat.edu.cn, ${ }^{\mathrm{c}}$ fhm@dhu.edu.cn, ${ }^{\mathrm{d}}$ ymkang@126.com
}

Keywords: fibrous filtration; single fiber; deposition morphologies; inertial impaction

\begin{abstract}
A three-dimensional stochastic simulation was developed to simulate growing process of particles deposit on a single fiber in Kuwarbara cell due to the collection mechanisms from inertial impaction and interception. The distributions of size of particle dendrites were calculated to quantificationally describe the complex deposit morphologies. The results indicate that the deposition morphologies strongly depend on Stokes number and interception parameter. A small stokes number tends to give a fewer but larger particle dendrites with a more open and loose structure, whereas a greater stokes number leads to a compact structure. The interception parameter shows different influencing degree to deposition morphology at different Stokes numbers. In the case of low Stokes number, there is no significant difference in heights of particle dendrites between various interception parameters, whereas for high Stokes number, the interception parameter plays a significant role in the morphologies transition from compact structures to dendritic. In addition, a larger interception parameter may weaken the effect of particle inertia on formation and growth of particle dendrites.
\end{abstract}

\section{Introduction}

Fibrous air filter is a widely adopted and highly efficient method to capture respirable particles in personal protective equipment, to reduce air contaminants in clean rooms, for particulate emission control in various industrial situations and many more. When aerosol particles flow through a fibrous filter, some of the particles are captured on the surface of the fibers constituting the filtration medium, resulting in a formation of chainlike agglomerates known as "paticles dendrites". These dendritic structures, in turn, cause a continuing change in the medium structure, leading an increase of the pressure drop cross filtration medium as the filter medium becomes clogged. Therefore, rational design, optimization, operation and innovation require the knowledge of a dynamics of dendrite growth and relevant important information about such deposition structure. The comprehensive theoretical model of dendritic deposition of submicron particles on a single fiber was first proposed by Payatakes and Gradoń [1]. In their work, only interception effect was considered to be collection mechanism for simplicity. Later on, Payatakes and Gradon extended this model to include other collection mechanisms such as inertial impaction, diffusion and electrostatic force [2,3]. More realistic structures of dendrites on a single fiber in Kuwabara's cell in regimes of inertial impaction and diffusion were obtained from the Monte Carlo simulations by Kanaoka, Emi, and Myoja [4,5], based on control surface concept and calculations for particles trajectories. Bahners et al. also used a similar stochastic simulation to estimate the dendritic deposition on parallel cylindrical fibres, but present only qualitative pictures of deposits.

Recently, some new numerical method (e.g., lattice-Boltzmann methods, boundary element and Fluent code based on finite element) have been used to simulate particle deposit formation on filter fibers, taking into account the two-way coupled particle-fluid [6-9]. However, this approach forbids the use of large numbers of particles due to its excessive computational requirement, thus being limited to treating small amounts of depositing particles or the case of two dimensions. In order to 
obtain some morphological properties that characterize large scale features of particles dendrties, a considerable number of deposited particles have to considered in three dimensional simulation.

The present study, we extended the previous simulation studies by Kanaoka et al using a three dimensional off lattice Monte Carlo method so that the particle deposition in fiber media may be simulated more realistically. However, no consideration of the complex interactions between particles and fliud is made for the reasons mentioned above. In addition, the relationship between the deposition mechanisms (inertial impaction and interception) and the relevant morphologies characteristics of the deposit was investigated in terms of the distribution of size of particle dendrites.

\section{Modeling growth of particles dendrites}

As shown in Fig. 1, the single fiber in Kuwabara cell will be adopted for analyzing growing process of particles dentrites on fibrous filtration medium. In this model one fiber collector of diameter df is placed centrally in a unit cell. A control surface (or release surface) is set far upstream from the fiber with its center perpendicularly located on x-axis. This control surface is considered as the reference plane from which trajectories of approaching particles originate, and the initial positions of each approaching particle is assigned by the random numbers within the range from 0 to 1 . Once the flow field around the collector is known and the initial positions of approaching particle is assigned, the trajectories of the approaching particle can be calculated by applying Newton's second law including inertial and aerodynamic forces. From the trajectory of the approaching particle, one can then determine whether this particle will be deposited onto the surfaces of a collector or onto the previously deposited particle. If a particle is collected by collectors (fiber or previously deposited particle), or move beyond unit cell, then this particle is discarded from the simulation and a new particle is released from control surface.

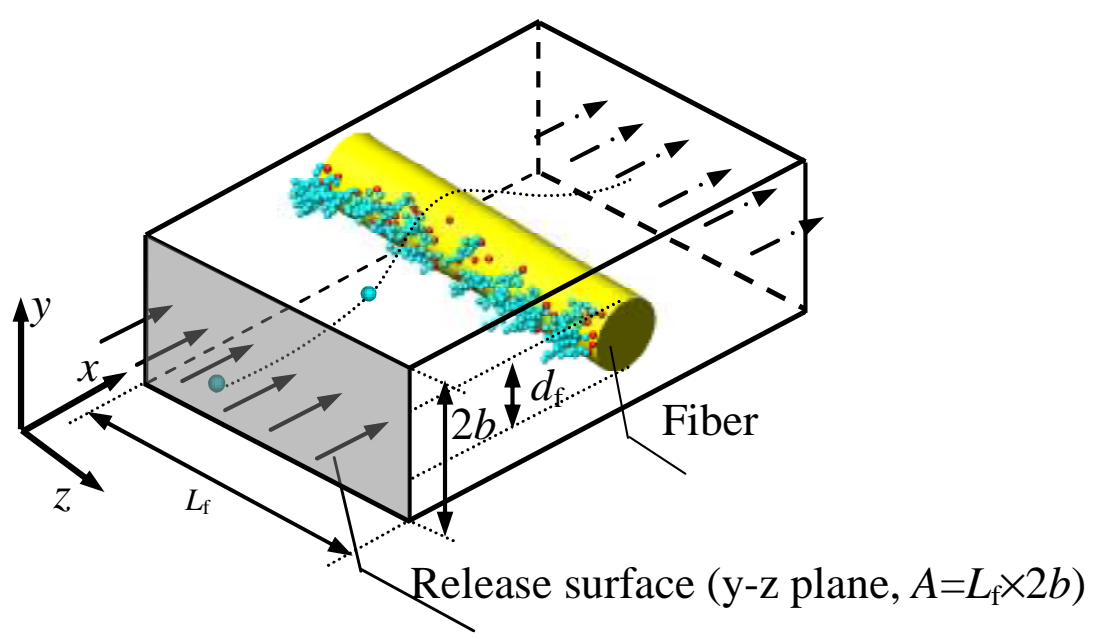

Fig. 1. Schematic of the three dimensional simulation of particle dendrites on a single fiber

\subsection{Kuwabara flow field}

In the present study, Kuwabara's model is adopted to describe the flow field around the collector. Generally, Kuwabara's model is identical to Happel's model except that velocity of flow field vanishes at the boundary of the fluid envelope $\mathrm{r}=\mathrm{b}$ around the collector, and the envelope's radius $\mathrm{b}$ can be defined as $b=R_{\mathrm{f}} c^{-1 / 2}$, where $R_{\mathrm{f}}$ is the radius of the collector and $c$ is the packing density of the filter bed (see in Fig1). Components of gas velocity for Cartesian co-ordinate system $\left(U_{x}, U_{y}\right)$ have a form [10]

$$
U_{x}=\frac{\hat{U}}{2 \mathrm{Ku}}\left\{2 \ln \frac{r}{R_{\mathrm{f}}}+\left(x^{2}+y^{2}\right) \cdot\left[\frac{R_{\mathrm{f}}{ }^{2}}{r^{4}}\left(1-\frac{c}{2}\right)-\frac{1}{r^{2}}\right]\right\}+\frac{\hat{U}}{2 \mathrm{Ku}}\left[c-\frac{c}{2{R_{\mathrm{f}}}^{2}}\left(x^{2}+3 y^{2}\right)\right]
$$




$$
U_{y}=-\frac{\hat{U}}{\mathrm{Ku}}\left[\frac{1}{r^{2}}-\frac{R_{\mathrm{f}}{ }^{2}}{r^{4}}+\frac{1}{2}\left(\frac{R_{\mathrm{f}}{ }^{2}}{r^{4}}-\frac{1}{R_{\mathrm{f}}{ }^{2}}\right)\right] x y
$$

where

$c=$ packing densityKu $=$ Kuwabara hydrodynamic factor $=-0.5 \ln ^{c}+c-0.5 c^{2}-0.75$

$r=$ a cylindrical coordinate

$x, y=$ Cartesian coordinate

$U_{\infty}=$ undisturbed approach velocity of air

$\hat{U}=$ mean velocity inside the filter $=U_{\infty} /(1-c)$.

\subsection{The Langevin trajectory equation}

In the present paper, only the interception and inertial impaction are considered, if the gravity and electric forces are ignored, the Langevin trajectory equation describing the balance on a spherical particle with a diameter $D_{\mathrm{p}}$ is written as

$\frac{1}{6} \pi \rho_{p} d_{p}^{3} \frac{\mathrm{d}}{\mathrm{d} t}\left(\stackrel{\mathrm{V}}{U}-\stackrel{\mathrm{V}}{V_{p}}\right)=\sum_{i} \stackrel{\mathrm{V}}{F_{i}}$

where $m_{\mathrm{p}}$ is the mass of the particle, $\mathrm{V}$ is the particle velocity vector, $\mathrm{t}$ is the time and $\mathbf{F}_{\mathrm{d}}$ is the drag force which can be expressed by $\mathbf{F}_{\mathrm{d}}=3 \pi \mu D_{\mathrm{p}}(\mathrm{u}-\mathrm{v}) / C_{\mathrm{m}}$. By substituting the expression of the drag force shown in Eq.(11), the force balance in the $\mathrm{x}$-derection and the y-derection yield:

where $D_{\mathrm{p}}$ is particle diameter, $\rho_{\mathrm{p}}$ is particle density, $\mu$ is fluid viscosity, $t$ is time, $U_{x}$ and $U_{y}$ are air velocity components in $x$ and $y$ directions, respectively, $V_{x}$ and $V_{y}$ is particle velocity components in $x$ and $y$ directions, respectively.

For the convenience of numerical solution of Eq. (3), a set of dimensional quantities are introduced as follows

$$
x^{*}=\frac{x}{R}, y^{*}=\frac{y}{R}, t^{*}=\frac{U_{\infty} t}{R}, U_{x}^{*}=\frac{U_{x}}{U_{\infty}}, U_{y}^{*}=\frac{U_{y}}{U_{\infty}}
$$

In a cartesian coordinate system, applying above dimensional quantilties, the Eq. (3) may be rewritten in dimensionless components form in $x$ and $y$, respectively.

$$
\begin{aligned}
& S t \frac{\mathrm{d}^{2} x^{*}}{\mathrm{~d} t^{* 2}}+\frac{\mathrm{d} x^{*}}{\mathrm{~d} t^{*}}-U_{x}^{*}=0 \\
& S t \frac{\mathrm{d}^{2} y^{*}}{\mathrm{~d} t^{* 2}}+\frac{\mathrm{d} y^{*}}{\mathrm{~d} t^{*}}-U_{y}^{*}=0
\end{aligned}
$$

Where St is Stokes number, $\mathrm{St}=2 C_{\mathrm{m}} \rho_{\mathrm{p}} R_{\mathrm{p}}^{2} U_{\infty} / 9 \mu D_{\mathrm{f}}, D_{\mathrm{f}}$ is fiber diameter and $R_{\mathrm{p}}$ and $R_{\mathrm{f}}$ are the radius of particle and fiber, respectively.

Solving Eqs. (4) and (5) may determine the particle trajectories for different initial coordinates and different Stokes numbers.

\section{Results and Discussion}

A typical set of results is presented in Fig. 2, in which various view of the deposits are shown. It is observed that the deposition morphologies strongly depend on Stokes number, St, which measured the domination of inertial impaction. Generally, a small stokes number tend to give a fewer but larger particle dendrites with a more open and loose structure, whereas a greater stokes number leads to a compact structure $(\mathrm{St}=2)$. Moreover, these dendrites are lack of regularity in morphologies, it is necessary to use a statistical means to quantificationally describe the deposit morphologies. For the purpose of illustration, the size distribution of particle dendrites were adopted to describe deposit 
morphology and its evolution on the basis of the average results of a large of simulations at the same set of filtration conditions. The sizes of particle dendrites may be classified by grouping dendrites in a manner shown in Table1, which is similar to that used by Jung and Tien (1993) in their simulations of aerosol deposition on granular media [11], and the fractional contributions of each group of dendrites to the extent of deposition may be to estimated in terms of the ratio of the number or volume of particles contained in each group of dendrites to the total number of particles deposited.

Table 1 Classification of particle dendrites

\begin{tabular}{llll}
\hline $\begin{array}{l}\text { dendrite type } \\
\zeta\end{array}$ & $\begin{array}{l}\text { Number of particles present in the } \\
\text { dendrite }\end{array}$ & $\begin{array}{l}\text { dendrite type } \\
\zeta\end{array}$ & $\begin{array}{l}\text { Number of particles } \\
\text { present in the dendrite }\end{array}$ \\
\hline 1 & 1 & 11 & $26-30$ \\
2 & 2 & 12 & $31-35$ \\
3 & 3 & 13 & $36-40$ \\
4 & 4 & 14 & $41-50$ \\
5 & 5 & 15 & $51-60$ \\
6 & $6-8$ & 16 & $61-80$ \\
7 & $9-10$ & 17 & $81-100$ \\
8 & $11-15$ & 18 & $101-150$ \\
9 & $16-20$ & 19 & $151-200$ \\
10 & $21-25$ & 20 & $200-500$ \\
\hline
\end{tabular}

The distributions of size of particle dendrites as function of the extent of deposition (namely, the number of particles deposited on fiber surface, $N_{\mathrm{c}}$ ) as shown in Fig. 3. For the case of $\mathrm{St}=0$, It can see that the majority of dendrites contain less than six particles at earlier deposition stage. As the number of deposited particles increase up to five hundreds, some dendrites as larger as having more than two hundreds particles (i.e., dendrite type is 20) were observed, which means that the approaching particles can be collected very effectively due to significant interception effect from the huge particle dendrites in such condition. However, for the case of $\mathrm{St}=2$ where particles have relatively higher inertia when moving towards collector, the dendrites having one to two particles are predominant at earlier deposition stage, and few large dendrites having more than twelve particles was found even at higher degree of particle deposition $\left(N_{\mathrm{c}}=500\right)$. Therefore, ones may expect that the heavier particles with higher inertia are more likely to form many but small dendrites on fiber surface, and resulting more dense packed structure, whereas the particles with lower density tend to form a fewer but larger particle dendrites at the same amount of deposited particles and filtration velocity.

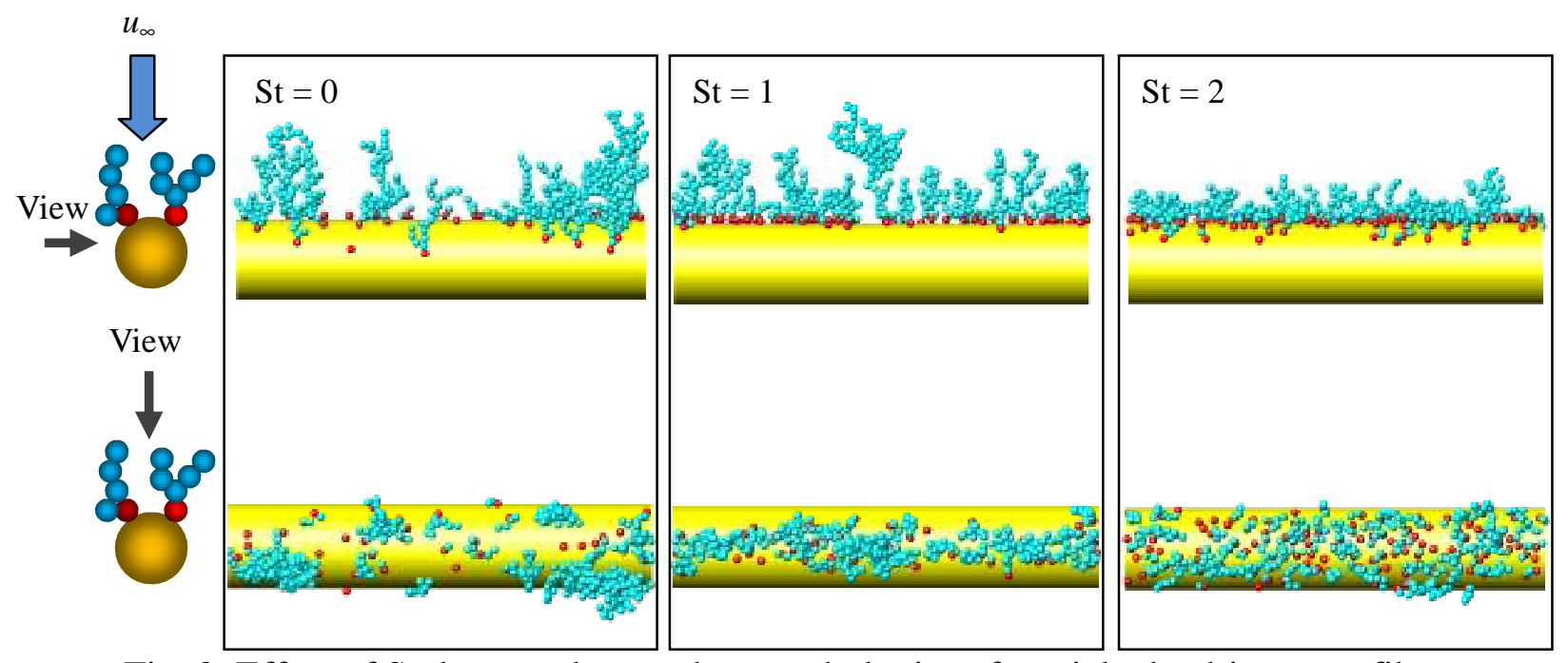

Fig. 2. Effect of Stokes number on the morphologies of particle dendrites on a fiber. 

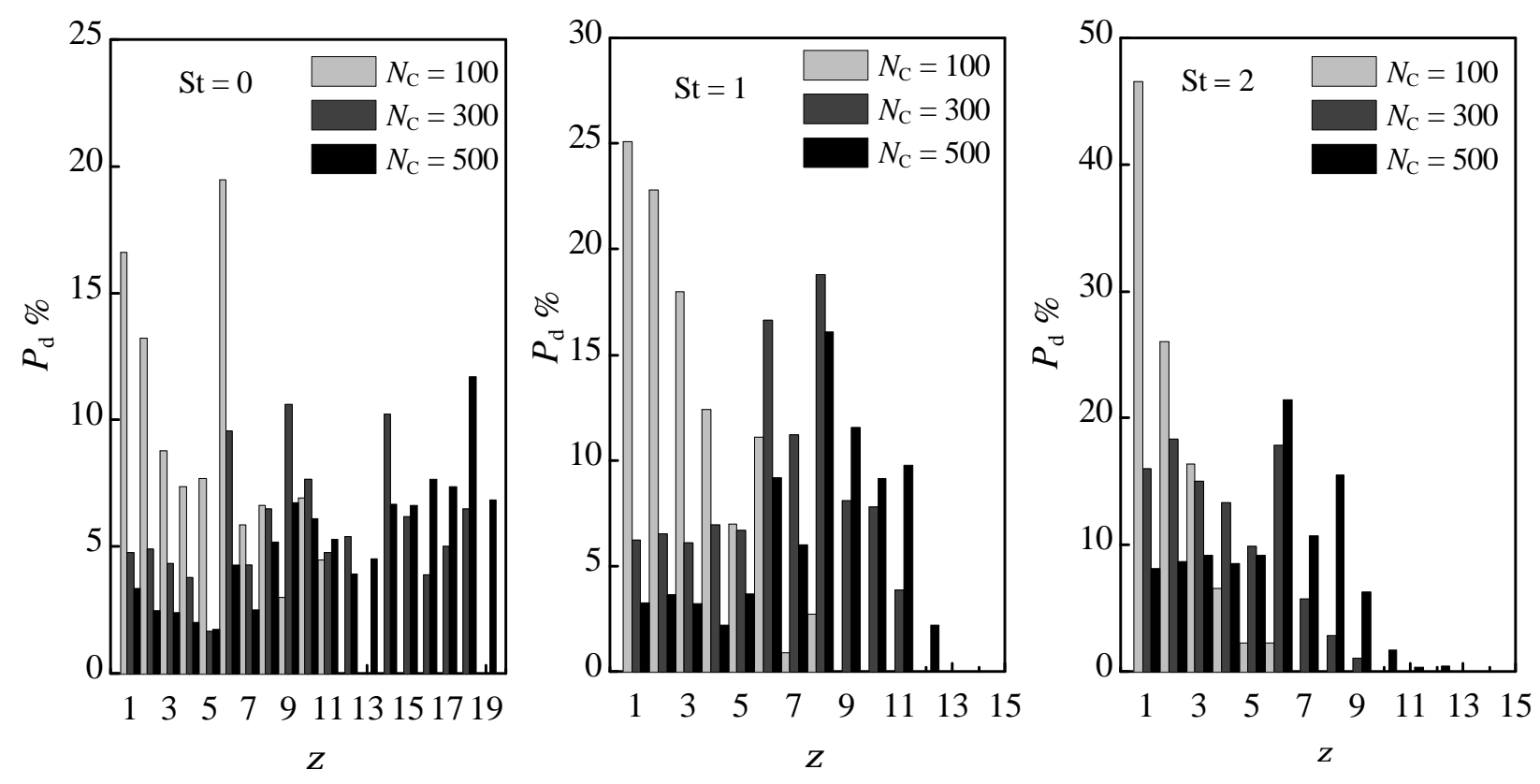

Fig. 3. Contributions of different particle dendrite type to total extent of deposition for different St at different deposition amount. $\left(d_{\mathrm{f}}=10 \mu \mathrm{m}, L_{\mathrm{f}}=50 \mu \mathrm{m}, d_{\mathrm{p}}=1 \mu \mathrm{m}, \alpha=0.03\right)$.

The effect of interception on the morphologies of deposit is shown in Fig. 4. In case of $\mathrm{St}=0$, it can not seem that the marked differences in the morphologies of particle dendrites amonge three interception parameters (i.e., $N_{\mathrm{R}}=0.1, N_{R}=0.2$ and $N_{R}=0.5$ ), and these dendrites have almost same morphologies, which are quite close to the experimental observations by Kasper et al. (see Fig. 6 in their paper, 2010) [12]. As for a higher stokes number $(\mathrm{St}=2)$, it is clear that an increase in interception causes a more open and loose particles dendrites in shape. It should note that when $N_{R}=$ 0.5 , considered to be the case of high interception parameter, the resulting deposition structures have rather similar heights and widths regardless of the magnitudes of Stokes numbers. These results indicate that an increase in interception parameter is possible to weaken the influence of particle inertia on formation and growth of particle dendrites on a fiber surface.
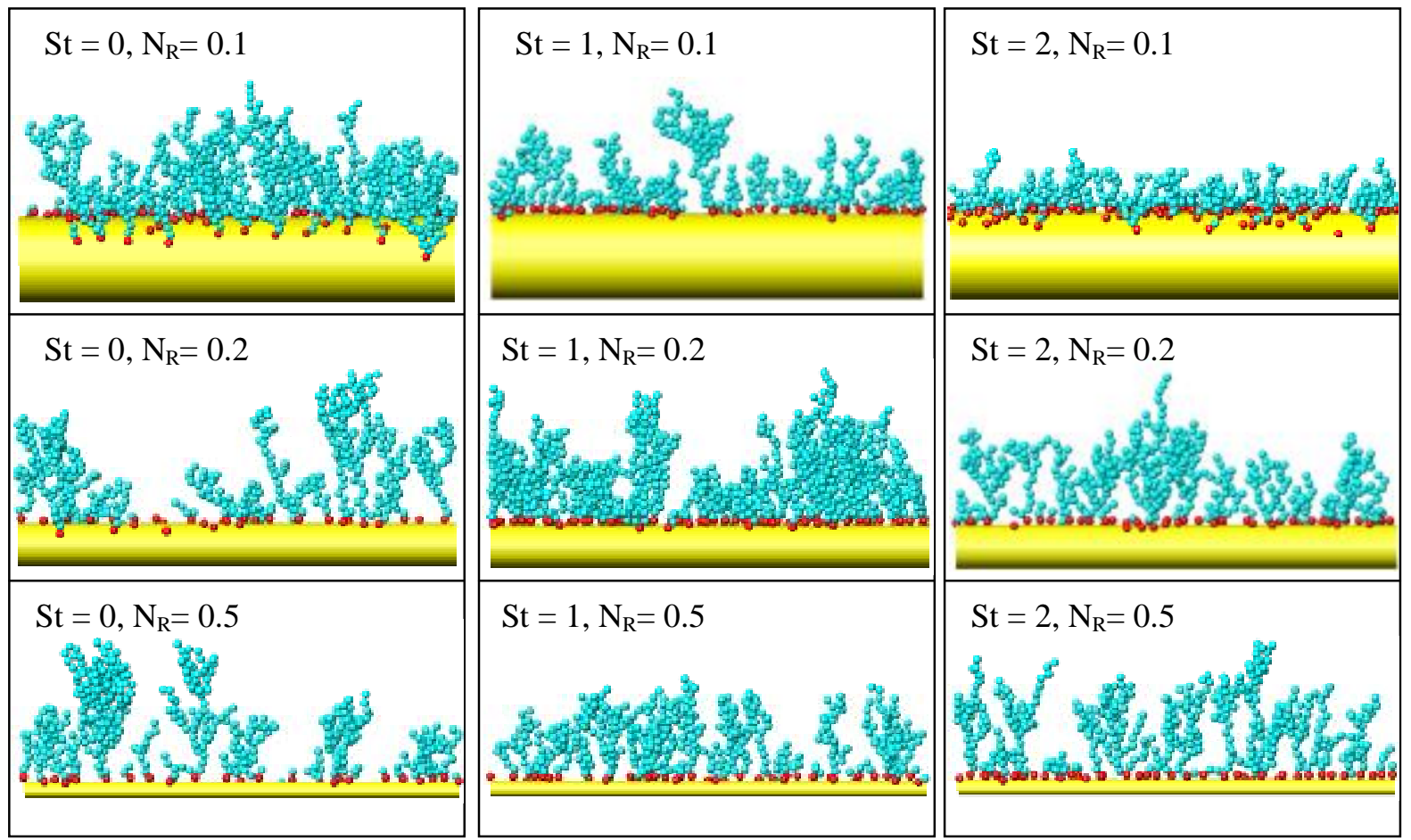

Fig. 4. Effect of interception parameter on the deposition morphologies on a fiber. 
The results of the size distributions of particle dendrites in Fig. 5 further confirm that the interception parameter have different influencing degree to deposition morphology for different Stokes numbers. For example, when $\mathrm{St}=0$, various types of particle dendrites formed on fibers with different diameters have similar contributions to the total number of deposited particles, whereas at higher Stokes number (i.e., St $=2$ ), the particle deposition tend to form larger dendrites with increasing $\mathrm{Nr}$.
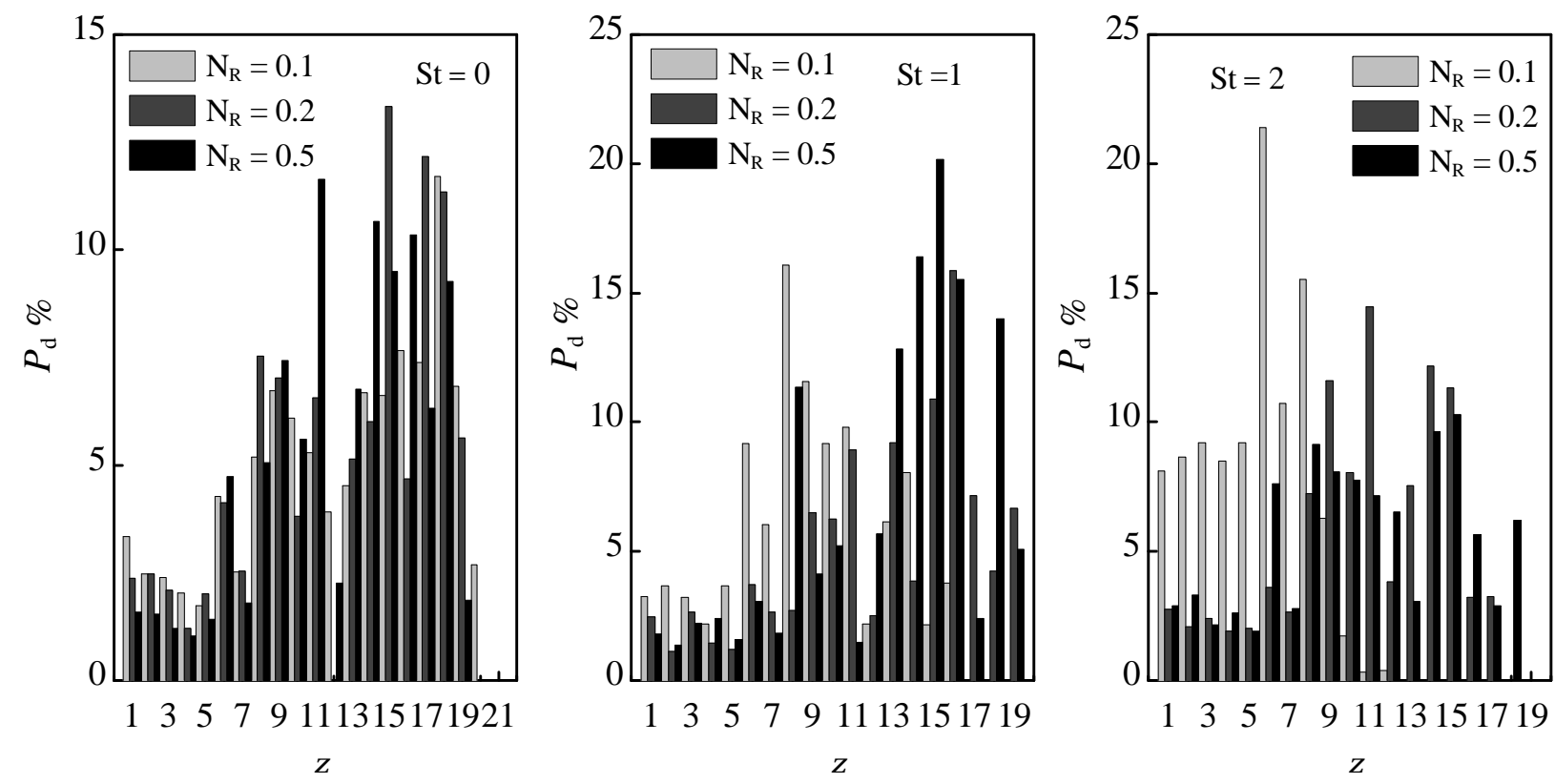

Fig. 5. Contributions of different particle dendrite type to total extent of deposition for different $N_{\mathrm{R}}$ at different Stokes number. $\left(L_{\mathrm{f}}=50 \mu \mu, d_{\mathrm{p}}=1 \mu \mathrm{m}, \alpha=0.03\right)$

\section{Conclusions}

In the present study, a three-dimensional stochastic simulation was developed to obtain useful insights into morphology of the deposit in the regime of inertial impaction and interception based on the information of the position of the deposited particles. The simulation results show that fairly good agreement in morphology of particle dendrites was obtained with experimental observations by Kasper et al without particles rebound behavior, and offer a useful information for a further understanding for particle deposition in fibrous media.

\section{Acknowledgements}

This work was financially supported by the National Natural Science Foundation of China (51178094) and Natural Science Foundation of Guilin University of Aerospace Technology (YJ1305).

\section{References}

[1] A.C. Payatakes, C.Tien, Particle deposition in fibrous media with dendrite-like pattern: a preliminary model, Journal of Aerosol Science 7(1976): 85-100.

[2] A.C. Payatakes, L. Gradoń, Dendritic deposition of aerosol particles in fibrous media by inertial impaction and interception, Chemical Engineering Science 35(1980): 1083-1096.

[3] A.C. Payatakes, L. Gradoń, Dendritic deposition of aerosols by convective Brownian diffusion for small, intermediate and high particle Knudsen numbers, AIChE Journal 26(1980): 443-454. 
[4] C. Kanaoka, H. Emi, T. Myojo, Simulation of the growing process of a particle dendrite and evaluation of a single fiber collection efficiency with dust load, Journal of Aerosol Science 11 (1980): 377-389.

[5] M. Wongsri, W. Tanthapanichakoon, C. Kanaoka, et al., Convective diffusional collection of polydisperse aerosols on a dust loaded fiber, Advanced Powder Technology 2 (1991): 11-23.

[6] H. Wang, H. Zhao, K. Wang, et al., Simulation of filtration process for multi-fiber filter using the Lattice-Boltzmann two-phase flow model, Journal of Aerosol Science 66 (2013): 164-178.

[7] S.A. Hosseini, H.V. Tafreshi, Modeling particle-loaded single fiber efficiency and fiber drag using ANSYS-Fluent CFD code, Computers and Fluids 66 (2012): 157-166.

[8] F. Qian, N. Huang, X. Zhu, et al., Numerical study of the gas-solid flow characteristic of fibrous media based on SEM using CFD-DEM, Powder Technology 249 (2013): 63-70.

[9] S.Q. Li, J.S.Marshall, Discrete element simulation of micro-particle deposition on a cylindrical fiber in an array, Journal of Aerosol Science 38 (2007): 1031-1046.

[10]S. Kuwabara, The forces experienced by randomly distributed parallel circular cylinders or spheres in a viscous flow at small Reynolds numbers, Journal of the physical society of Japan 14 (1959): 527-532.

[11] Y. Jung, C. Tien, Simulation of aerosol deposition in granular media, Aerosol science and technology18 (1993): 418-440.

[12] G. Kasper, S. Schollmeier, J. Meyer, Structure and density of deposits formed on filter fibers by inertial particle deposition and bounce, Journal of Aerosol Science 41 (2010): 1167-1182. 